\title{
Breast Microglandular Adenosis
}

National Cancer Institute

\section{Source}

National Cancer Institute. Breast Microglandular Adenosis. NCI Thesaurus. Code C5199.

A rare variant of breast adenosis characterized by the proliferation of small round glands in a collagenous stroma. The epithelial cells are cuboidal and there are no myopepithelial cells present. There is no evidence of atypia. 